\title{
THE CENTER OF THE FREE PRODUCT OF DISTRIBUTIVE LATTICES ${ }^{1}$
}

\section{RAYMOND BALBES}

Abstract. The object of this paper is to show that for members $L$ and $M$ in the class of distributive lattices with zero and unit, the center of the free product of $L$ and $M$ is the free product of their centers.

We can assume that $L$ and $M$ are subalgebras (that is $(0,1)$ sublattices) of their free product, which we denote by $L * M$. This means [2] that $L \cup M$ generates $L * M$ and for $a_{1}, a_{2} \in L, b_{1}, b_{2} \in M$ :

$$
a_{1} b_{1} \leqq a_{2}+b_{2} \text { implies } a_{1} \leqq a_{2} \text { or } b_{1} \leqq b_{2} .
$$

Since the center $C(L)$ of $L$ is exactly its subalgebra of complemented elements $[1$, p. 67], we have immediately that $C(L) * C(M)$ $\subseteq L * M$. But an element $x=\sum_{i=1}^{p} a_{i} b_{i}$, where $p \geqq 1, a_{i} \in C(L), b_{i}$ $\in C(M)$, has a complement, namely $\prod_{i=1}^{p}\left(a_{i}^{\prime}+b_{i}^{\prime}\right)$, where $a_{i}^{\prime}$ is the complement of $a_{i}$ in $L$ and $b_{i}^{\prime}$ is the complement of $b_{i}$ in $M$. So $C(L)$ $* C(M) \subseteq C(L * M)$.

To prove the reverse inclusion, it suffices to show that if $x \in C(L * M)$ and

$$
x=\sum_{i=1}^{p} a_{i} b_{i}, \quad p \geqq 1, \quad a_{i} b_{i} \neq 0, \quad a_{i} \in L, \quad b_{i} \in M,
$$

then any $a_{i}$ that appears in (2) can be replaced by a member of $C(L)$ and still leave (2) valid. Indeed, if this replacement is possible then each $a_{i}$ can be successively replaced by members of $C(L)$ and then the whole process repeated for each $b_{i}$; thus showing that $x \in C(L) * C(M)$.

Now to prove that this replacement is possible suppose $x \neq 0,1$ has the representation in (2) and that $x^{\prime}$ is the complement of $x$ in $C(L * M)$. So

$$
x^{\prime}=\sum_{j=1}^{q} \alpha_{j} \beta_{j} \quad \text { for some } q \geqq 1, \alpha_{j} \in L, \beta_{j} \in M .
$$

Received by the editors October 5, 1970.

A MS 1969 subject classifications. Primary 0635, 0640; Secondary 0805, 0810.

Key words and phrases. Free product, center, complemented elements, zero, unit.

1 The research and preparation of this paper was supported in part by NSF GP 11893.

Copyright (c) 1971, American Mathematical Society 
We will replace $a_{1}$ by an element of $C(L)$. If $a_{1} \in C(L)$ we are finished so suppose $a_{1} \notin C(L)$. Noting the convention $\sum \varnothing=0$ and the fact that (1) implies that for each $j \in\{1, \cdots, q\}$ either $a_{1} \alpha_{j}=0$ or $b_{1} \beta_{j}=0$, we have:

$$
\begin{gathered}
\left(a_{1}+\sum_{i=2}^{p} b_{i}\right)+\left(\sum\left\{\alpha_{i} \mid a_{1} \alpha_{j}=0\right\}+\sum\left\{\beta_{j} \mid b_{1} \beta_{j}=0\right\}\right) \\
\geqq x+x^{\prime}=1 .
\end{gathered}
$$

Now $a_{1} \sum\left\{\alpha_{j} \mid a_{1} \alpha_{j}=0\right\}=0$ so $a_{1}+\sum\left\{\alpha_{j} \mid a_{1} \alpha_{j}=0\right\} \neq 1$. Applying (1) to (3) we obtain $\sum_{i=2}^{p} b_{i}+\sum\left\{\beta_{j} \mid b_{1} \beta_{j}=0\right\}=1$. This implies

$$
b_{1} \leqq \sum_{i=2}^{p} b_{i}
$$

Let $\left\{S_{1}, \cdots, S_{r}\right\}$ be all subsets $S$ of $\left\{b_{2}, \cdots, b_{r}\right\}$ with the property that $b_{1} \leqq \sum(S)$. Note that from (4) and (2), $r \geqq 1$ and $S_{j} \neq \varnothing$, for $j=1, \cdots, r$. Set $T_{j}=\left\{a_{k} \mid b_{k} \in S_{j}\right\}$ for $j=1, \cdots, r$. We will show that the required replacement for $a_{1}$ is $A=a_{1}+\prod\left(T_{1}\right)+\cdots+\prod\left(T_{r}\right)$. Clearly $x \leqq A b_{1}+a_{2} b_{2}+\cdots+a_{p} b_{p}$. On the other hand, for each $j \in\{1, \cdots, r\}, \Pi\left(T_{j}\right) b_{1} \leqq \prod\left(T_{j}\right) \sum\left(S_{j}\right) \leqq \sum\left\{a_{k} b_{k} \mid b_{k} \in S_{j}\right\} \leqq x$. So $A b_{1} \leqq x$ and

$$
x=A b_{1}+a_{2} b_{2}+\cdots+a_{p} b_{p} .
$$

It remains to prove $A \in C(L)$. Let $J$ be the family of all sets $T$ which consist of exactly one member from each of the sets $T_{1}, \cdots, T_{r}$. For such a $T \in J,(5)$ yields

$$
\begin{aligned}
(A+ & \left.\sum(T)+\sum\left\{b_{i} \mid a_{i} \notin T, i \geqq 2\right\}\right) \\
& +\left(\sum\left\{\alpha_{j} \mid A \alpha_{j}=0\right\}+\sum\left\{\beta_{j} \mid b_{1} \beta_{j}=0\right\}\right) \geqq x+x^{\prime}=1 .
\end{aligned}
$$

Now if $\sum\left\{b_{i} \mid a_{i} \notin T, i \geqq 2\right\}+\sum\left\{\beta_{j} \mid b_{1} \beta_{j}\right\}=1$ then

$$
b_{1} \leqq \sum\left\{b_{i} \mid a_{i} \notin T, i \geqq 2\right\} \quad \text { so } \quad\left\{b_{i} \mid a_{i} \notin T, i \geqq 2\right\}=S_{j}
$$

for some $j \in\{1, \cdots, r\}$. But this is impossible, for by the definition of $T$, there is a member $a_{i_{0}} \in T_{j} \cap T$, where $i_{0} \geqq 2$, so

$$
b_{i_{0}} \in S_{j}-\left\{b_{i} \mid a_{i} \notin T, i \geqq 2\right\}=\varnothing .
$$

By applying (1) to (6) we obtain for each $T \in J$ :

$$
A+\sum(T)+\sum\left\{\alpha_{j} \mid A \alpha_{j}=0\right\}=1 .
$$

Thus, 


$$
\begin{aligned}
1 & =A+\prod\left\{\sum(T) \mid T \in J\right\}+\sum\left\{\alpha_{j} \mid A \alpha_{j}=0\right\} \\
& =A+\left(\prod\left(T_{1}\right)+\cdots+\prod\left(T_{r}\right)\right)+\sum\left\{\alpha_{j} \mid A \alpha_{j}=0\right\} \\
& =A+\sum\left\{\alpha_{j} \mid A \alpha_{j}=0\right\}
\end{aligned}
$$

This means that $A$ and $\sum\left\{\alpha_{j} \mid A \alpha_{j}=0\right\}$ are complements in $L$, which completes the proof.

\section{REFERENCES}

1. G. Birkhoff, Lattice theory, 3rd ed., Amer. Math. Soc. Colloq. Publ., vol. 25, Amer. Math. Soc., Providence, R.I., 1967. MR 37 \#2638.

2. G. Grätzer and H. Lakser, Chain conditions in the distributive free product of lattices, Trans. Amer. Math. Soc. 144 (1969), 301-312. MR 40 \#176.

3. R. Sikorski, Products of abstract algebras, Fund. Math. 39 (1952), 211-228. MR $14,839$.

University of Missouri at St. Louis, St. Louis, Missouri 63121 